\title{
Acoustic radiation efficiency of a periodically corrugated rigid piston
}

\author{
Héctor Estrada, ${ }^{1,2, a)}$ Antonio Uris, ${ }^{1}$ and Francisco Meseguer ${ }^{1,2}$ \\ ${ }^{1}$ Centro de Tecnologías Físicas, Unidad Asociada ICMM-CSIC/UPV, Universitat Politècnica de València, \\ Av. de los Naranjos $s / n, 46022$ Valencia, Spain \\ ${ }^{2}$ Instituto de Ciencia de Materiales de Madrid (CSIC), Cantoblanco, 28049 Madrid, Spain
}

(Received 26 July 2012; accepted 16 August 2012; published online 5 September 2012)

\begin{abstract}
The radiation of sound by a periodically corrugated rigid piston is explored using theoretical and numerical approaches and compared with the radiation of flat rigid piston. The depth and the period of the corrugation are considered to be comparable with the wavelength in the surrounding fluid. Radiation enhancement is predicted due to cavity resonances and coherent diffraction. In addition, broad regions of low radiation efficiency are observed. Both effects could have an impact in acoustic transducers technology, either to increase the piston radiated power or to create a source of evanescent acoustic waves. The possibilities offered by this strategy in the nonlinear acoustic regime are also briefly discussed. (c) 2012 American Institute of Physics.

[http://dx.doi.org/10.1063/1.4748868]
\end{abstract}

The radiation of acoustic waves is used in a wide range of different applications ranging from biomedical ones to non-destructive testing. In any of these systems, the transducer is a crucial element. The performance of such a system is often limited by the transducer acoustic radiation properties. In addition, the behavior of an acoustic transducer is influenced by many factors, being its mechanical construction the most direct one. ${ }^{1}$

Nowadays, the piston radiator is maybe the most important radiation source in ultrasonics and its radiation properties constitute a classical-physics problem. However, besides the great advancement in transducer arrays and beam steering, ${ }^{2,3}$ piezo-composite materials, ${ }^{4}$ capacitive micromachined transducers, ${ }^{5}$ and matching layers, ${ }^{6}$ very little has been done concerning the radiating surface of the transducer.

On the other hand, recent advances in the study of periodically corrugated surfaces and perforated plates, mainly motivated from interesting results from optics, have shown exotic properties that could be used for modifying the propagation of elasto-acoustic waves. Most of these studies concentrate on the transmission and reflection properties of perforated/corrugated plates, as a direct translation of systems being studied in optics. ${ }^{7}$ Resonant corrugated panels have been also considered for sound insulation purposes. ${ }^{8}$ However, no study has been done considering how a corrugated surface will affect the radiation of acoustic waves in a transducer, despite its technological relevance.

In this letter, we present a theoretical and numerical study on the effect of a rigid periodically corrugated surface in the radiation of acoustic waves compared with the classical rigid piston. The depth and the period of the corrugation have the same order of magnitude than the wavelength in the fluid. Coherent diffraction and cavity resonances give rise to both radiation enhancement and radiation inhibition depending on the geometrical properties of the corrugation.

Given a fluid of density $\rho$ and sound speed $c$, we consider a perfectly rigid surface vibrating at a frequency $\omega$ with

\footnotetext{
${ }^{\text {a) }}$ Author to whom correspondence should be addressed. Electronic mail: hector.estrada.b@gmail.com.
}

velocity $v_{0}$ as depicted in Figs. 1(a) and 1(b). Solving the two-dimensional problem by means of the finite element method (Comsol Multiphysics) for a flat and a corrugated piston of the same size $D=9.6 \lambda(\lambda=2 \pi c / \omega)$, the latter having only ten cavities $\left(N_{c}=10\right)$, we obtain the fields depicted in Figs. 2(a) and 2(b). The color scale represents the time-averaged quadratic sound pressure $|p|^{2}$ in normalized units. Although both pistons are radiating with the same velocity amplitude $v_{0}$, the radiation enhancement provided by the corrugation is evident. More information can be obtained by looking at the normalized pressure level (NPL) along the $z$ direction across the pistons radiation axis (Fig. 2(c)). The pressure level is normalized by the maximum provided by the flat transducer. The classical result of the flat transducer, i.e., an oscillating nearfield reaching a maximum at $z / D \approx 3$ before decaying into the farfield, clearly contrasts with the behavior of the corrugated piston. The maximum NPL of the corrugated piston is right at its face, $15 \mathrm{~dB}$ above the flat piston maximum. As $z / D$ increases, the NPL radiated by the corrugated piston strongly decreases but it stops oscillating and starts to maintain a monotonic decay $6 \mathrm{~dB}$ above the flat piston curve.

In order to understand this radiation enhancement and explore some other effects, we developed two-and threedimensional models following the formulation described in (a)

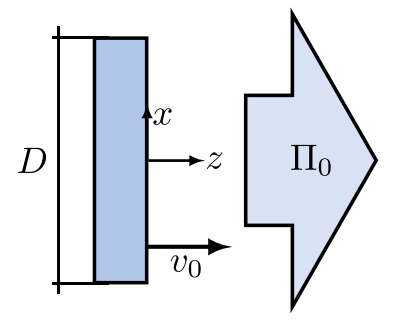

Flat (b)

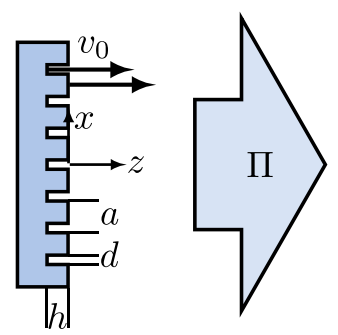

Corrugated
FIG. 1. Diagrams showing the geometry and the relevant variables and constants of the problem for (a) flat rigid piston and (b) for a periodically corrugated rigid piston. The radiated sound power is labeled as $\Pi_{0}$ and $\Pi$ for each case. 

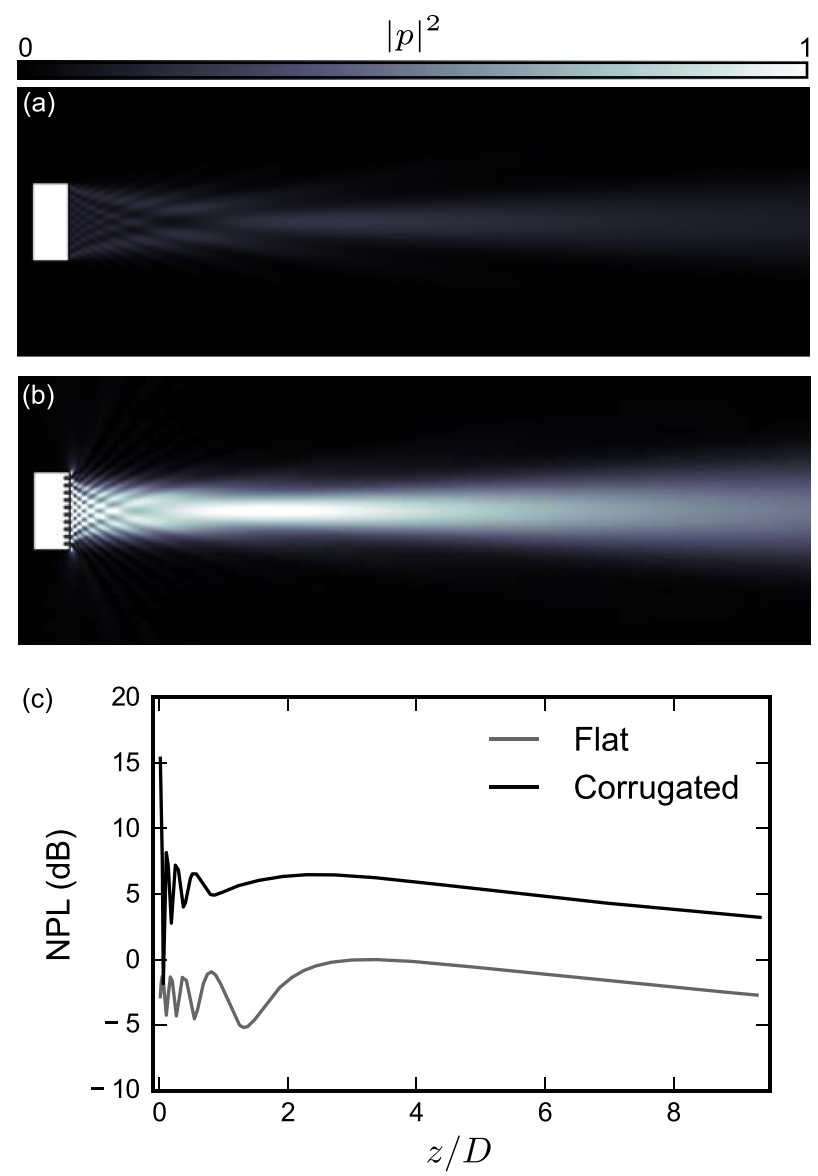

FIG. 2. Time averaged quadratic pressure field ( $|p|^{2}$ in normalized units) of a flat (a) and (b) a corrugated piston having ten cavities $\left(N_{c}=10\right), d=0.3 a$, and $h=0.71 a$ at a $\lambda=0.9$ (c) NPL in (dB) along $z / D$ at the radiation axis.

Ref. 9 for infinitely periodic structures. The sound pressure inside the cavity is expanded in terms of their eigenmodes $\phi_{m, n}\left(\mathbf{r}_{\|}\right)\left(\mathbf{r}_{\|}=(x, y)\right)$ for perfectly rigid boundaries as

$$
p_{c}=\sum_{m, n} \phi_{m, n}\left(\mathbf{r}_{\|}\right) \psi_{m, n}(z)
$$

where

$$
\psi_{m n}(z)=\left[\alpha_{m n}^{+} e^{\mathrm{i} q_{m n}(z+h)}+\alpha_{m n}^{-} e^{-\mathrm{i} q_{m n} z}\right],
$$

$q_{m n}=\sqrt{(\omega / c)^{2}-Q_{m n}^{2}}$, and $\alpha_{m n}^{ \pm}$corresponds to the wave amplitude coefficients. On the other hand, the sound pressure in the fluid surrounding the piston can be expanded as

$$
p_{\mathbf{G}}=\sum_{\mathbf{G}} \beta_{\mathbf{G}} \exp \left(\mathrm{i}\left(\mathbf{G r}_{\|}-q_{\mathbf{G}}(z+h)\right)\right) .
$$

The reciprocal lattice vector $\mathbf{G}$ depends on the array geometry and leads to $q_{\mathbf{G}}=\sqrt{(\omega / c)^{2}-|\mathbf{G}|^{2}}$. Then, imposing boundary conditions of fixed velocity $v_{0}$ at $z=-h$ in the cavity, continuity of pressure at the apertures entry, and continuity of velocity at $z=0$ yield a linear system of equations to be solved for $\alpha_{m n}^{ \pm}$and $\beta_{\mathbf{G}}$. Thus, the radiated sound power $\Pi$ in terms of $\beta_{\mathbf{G}}$ is given by

$$
\Pi=\frac{1}{8 \pi^{2} \omega \rho} \sum_{\mathbf{G}} \operatorname{Re}\left\{q_{\mathbf{G}}\right\}\left|\beta_{\mathbf{G}}\right|^{2} .
$$

As the corrugated piston is considered to be infinitely periodic, the radiation efficiency $\sigma=\Pi / \Pi_{0}$ is calculated considering an infinite flat piston whose radiated power is given by $\Pi_{0}=\rho c\left|v_{0}\right|^{2} / 8 \pi^{2}$. In addition, to allow a direct comparison with the finite element method, the radiated power has been calculated by integrating the time averaged intensity $I_{z}=\frac{1}{2} \operatorname{Re}\left\{p v_{z}^{*}\right\}\left(^{*}\right.$ means complex conjugate $)$ right at the piston surface with and without corrugation.

Before analyzing the phenomena, it is appropriate to test the consistency of the results among the different methods. Figure 3(a) shows the radiation efficiency as a function of $h / \lambda$ for a corrugation having $h / a=1.71$ and a hole filling fraction $f=0.3$ calculated by the modal model (2D and 3D, the latter for a square array of circular perforations) and the finite element method $\left(N_{c}=15,30\right)$. The agreement between the calculations demonstrates the suitability of $\sigma$ for the analysis. The effect of the finite piston size is particularly distinguishable at the rightmost dip, near the radiation enhancement peak.

Abrupt changes of $\sigma$ delimit low and near zero radiation efficiency regions, whose position is closely linked to the well known cavity resonances at $h / \lambda=(2 n+1) / 4$ for integer $n$. However, the closer the resonances are to the $\lambda=a$ point $(h / \lambda=1.71)$ the larger the shift toward lower frequencies, in agreement with previous results reported for perforated plates. ${ }^{10-13}$

To further clarify how the cavity resonances modify the radiation characteristics of the piston, Fig. 3(b) depicts the magnitude and the phase of $v_{z}$ right at the aperture entrance $(z=0)$. The abrupt phase changes from $\pi$ to $-\pi$ explain the dips in $\sigma$ and are localized near to a velocity maximum. The resonant origin of the velocity maxima is clear, in addition to
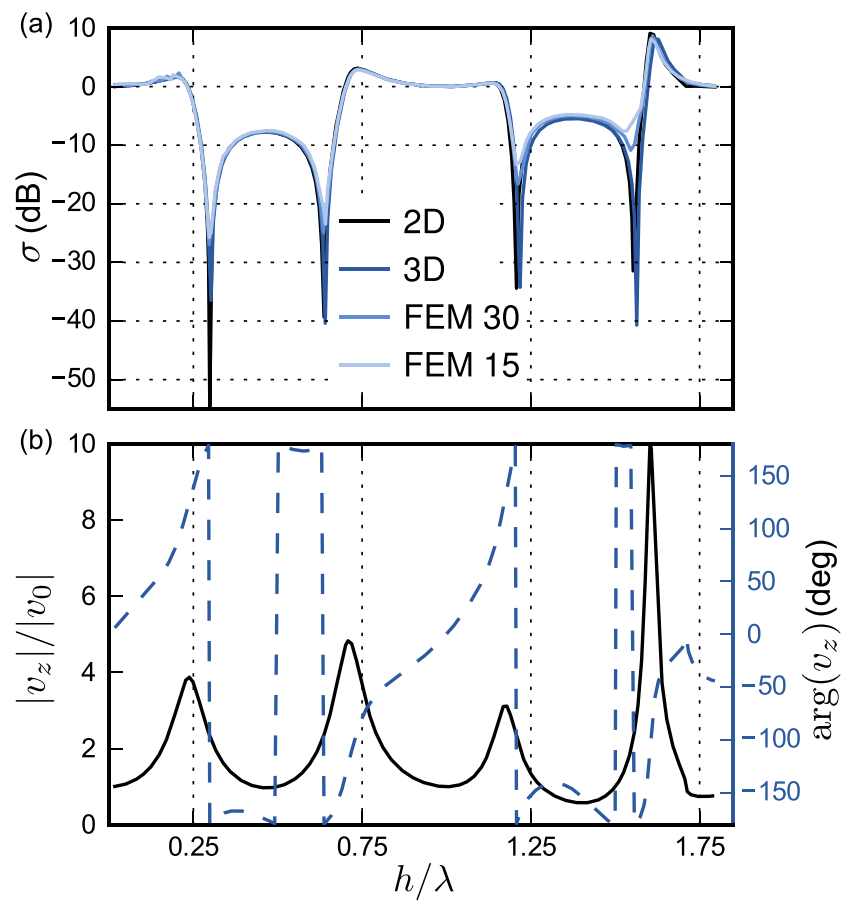

FIG. 3. (a) Radiation efficiency $\sigma$ in (dB) of a corrugated piston with proportions $h / a=1.71$ and a hole filling fraction $f=0.3$ calculated using different models (see labels) as a function of $h / \lambda$. (b) Magnitude (solid curve, left vertical axis) and phase (dashed curve, right vertical axis) of the average velocity at the aperture entrance calculated with the two-dimensional modal expansion as a function of $h / \lambda$. 
the important contribution of the array period to the 10 fold enhancement in $v_{z}$ at the high frequency peak. This is the mechanism that allows the radiation enhancement, i.e., a large increment in the particle velocity right at the piston surface. Of course, solely the magnitude of $v_{z}$ does not explain everything, because it interferes with the $v_{0}$ velocity of the piston rigid surface outside the cavity.

The next step is to observe how these radiation features vary with the geometrical parameters, which is represented in Fig. 4 calculated using our three dimensional modal model. By changing the $h / a$ ratio and keeping $f=0.3$, we obtain Fig. 4(a). As $\sigma$ is now plotted as a function of $a / \lambda$, the spikes vary their position. The existence of the radiation enhancement peak depends strongly on $h / a$. If the grooves are shallow enough, no radiation enhancement can be observed. This is in agreement with the analytical results reported in Ref. 14, where it has been shown that the array itself does not lead to any transmission enhancement without the fundamental contribution of the cavity resonances.

The hole filling fraction $f$ mostly influences the low radiation region, as can be seen from Fig. 4(b). The two radiation minima get close together as $f$ increases and reach eventually $\sigma<-20 \mathrm{~dB}$ before being fused in a broad minimum for $f=0.64$. However, this drastic drop in the radiation efficiency does not imply a decrease in the pressure amplitude at the corrugated piston surface. Figure 5(a) shows $\sigma$ as a function of $a / \lambda$ calculated using our three-dimensional model and the finite element method. Although for a low $f$ both theories agreed well, the finite size of the piston plays a role for high $f$ and low radiation efficiencies cannot be
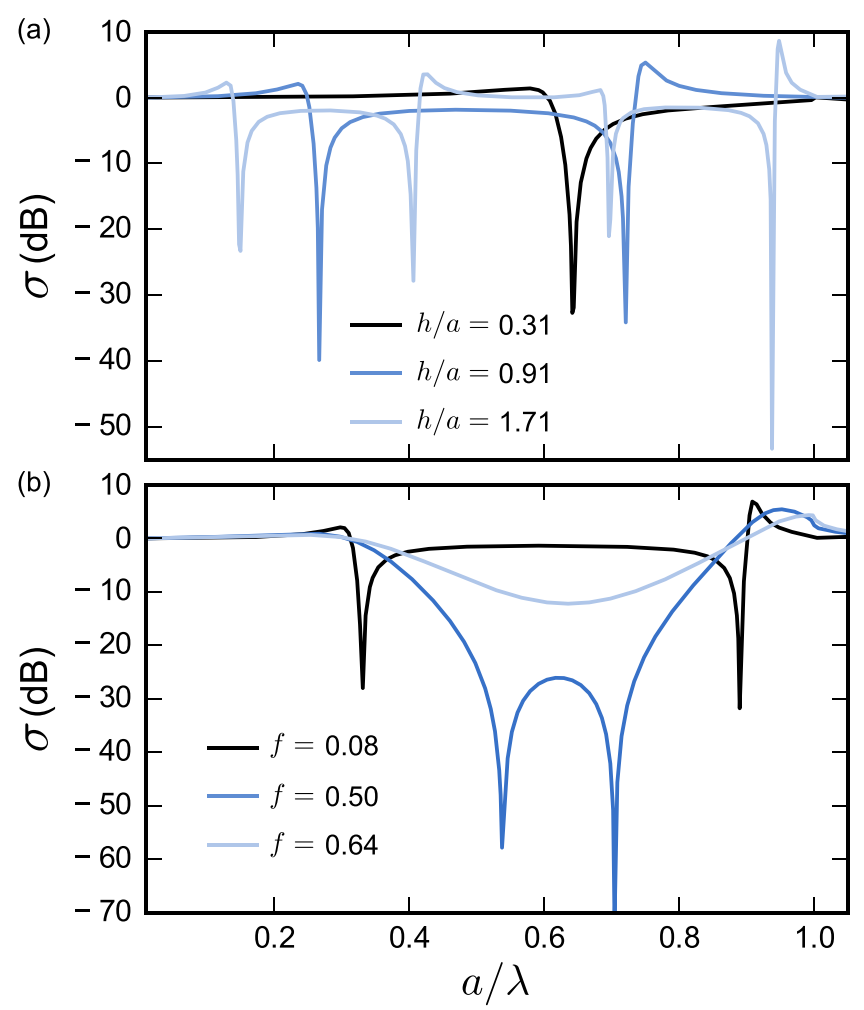

FIG. 4. Radiation efficiency $\sigma$ in $(\mathrm{dB})$ as a function of $a / \lambda$ of a corrugated piston (a) having $f=0.1$ for different $h / a$ ratios and (b) for fixed $h / a=0.71$ and different hole filling fractions $f$.
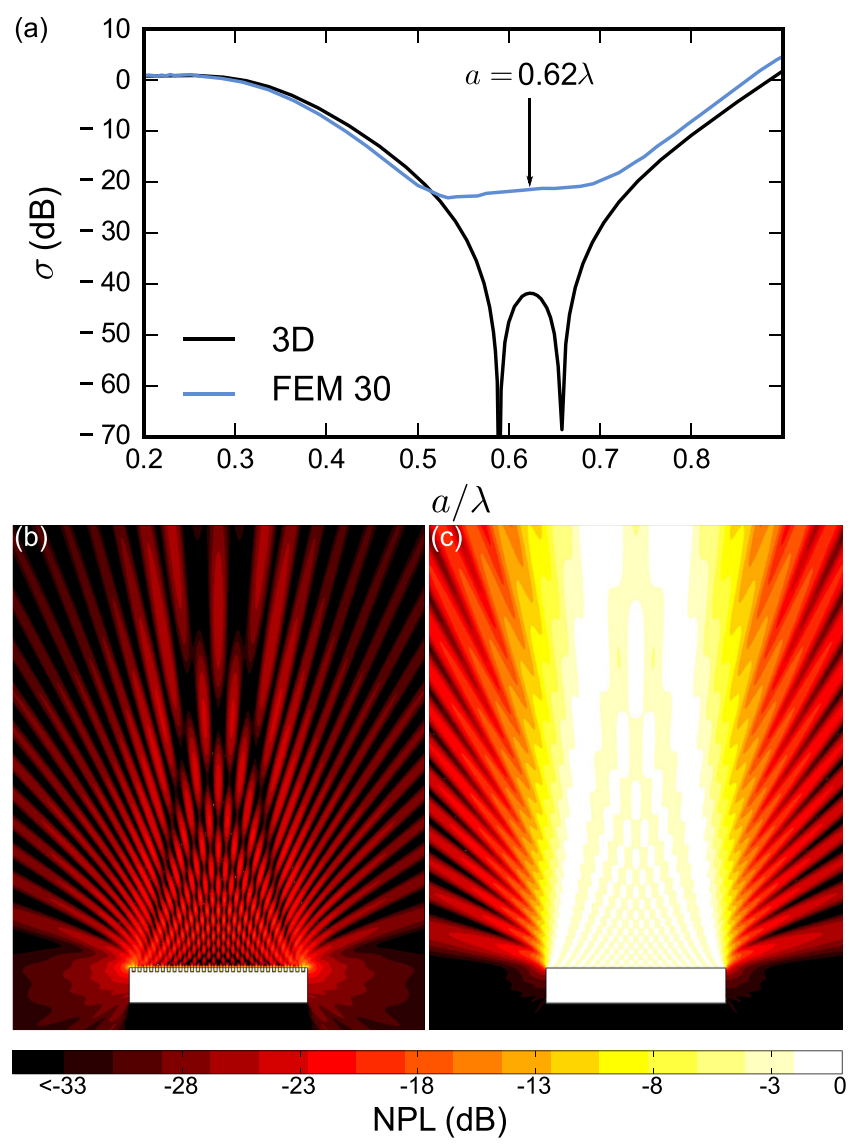

FIG. 5. (a) Radiation efficiency $\sigma$ in (dB) as a function of $a / \lambda$ for a corrugation having $h / a=0.71$ and $f=0.52$ calculated for infinite and finite pistons. The arrow shows the point at which the fields in (b) and (c) have been calculated. Normalized pressure level fields in $(\mathrm{dB})$ for a (b) corrugated and (c) flat piston at $a=0.62 \lambda$.

reached even for $N_{c}=30$. Despite the disagreement between the calculations methods, $-20 \mathrm{~dB}$ in radiation efficiency can still be distinguished from the pressure level fields of Figs. 5 (b) and 5(d) for $a=0.62 \lambda$. The pressure in the corrugated piston is concentrated at the corrugations and cannot be radiated. These radiation minima may be suitable for nearfield material inspection strategies using a corrugated piston as an evanescent acoustic source.

In our calculations, we did not include losses in the fluid. In practical implementations of this strategy for modulating the radiation, it will certainly constitute an important point to be considered. However, as far as the holes remain with a moderate depth to aperture size ratio $h / d$, losses should not affect the observation of the reported results neither in air nor in water. It is worth mentioning that due to the resonant features of the corrugated piston, the overall field maximum will be located right in front of the transducer (see Fig. 2(c)) and not far away as in flat pistons. Due to this, it would be interesting to test the effect of the corrugations in the nonlinear regime, where even for moderate amplitude the non linearities should appear already at the piston surface and thus, affect the whole radiation properties we have reported here and presumably differ from the classic piston behavior in the nonlinear regime.

This work has been supported by the Spanish MICINN (MAT2010-16879, Consolider CSD2007-00046 and Universitat 
Politecnica de Valencia (PAID-06-10-1839). We gratefully acknowledge the valuable help of Javier García de Abajo in the development of the modal model.

${ }^{1}$ C. Desilets, J. Fraser, and G. Kino, IEEE Trans. Sonics Ultrason. 25, 115 (1978).

${ }^{2}$ O. T. Von Ramm and S. W. Smith, IEEE Trans. Biomed. Eng. BME-30, 438 (1983).

${ }^{3}$ S. Smith, H. G. Pavy, Jr., and O. von Ramm, IEEE Trans. Ultrason. Ferroelectr. Freq. Control 38, 100 (1991).

${ }^{4}$ W. Smith, in Proceedings of IEEE Ultrasonics Symposium, 1989 (IEEE, 1989), p. 755, DOI: 10.1109/ULTSYM.1989.67088.

${ }^{5}$ O. Oralkan, A. Ergun, J. Johnson, M. Karaman, U. Demirci, K. Kaviani,

T. Lee, and B. Khuri-Yakub, IEEE Trans. Ultrason. Ferroelectr. Freq. Control 49, 1596 (2002).

${ }^{6}$ T. Gomez and F. Montero, IEEE Ultrason. Symp. 2, 1069 (2000).
${ }^{7}$ T. W. Ebbesen, H. J. Lezec, H. F. Ghaemi, T. Thio, and P. A. Wolff, Nature 391, 667 (1998).

${ }^{8} \mathrm{M}$. Hannink, "Acoustic resonators for the reduction of sound radiation and transmission," Ph.D. dissertation (University of Twente, Enschede, The Netherlands, 2007).

${ }^{9}$ H. Estrada, P. Candelas, A. Uris, F. Belmar, F. Meseguer, and F. J. García de Abajo, Wave Motion 48, 235 (2011).

${ }^{10}$ B. Hou, J. Mei, M. Ke, W. Wen, Z. Liu, J. Shi, and P. Sheng, Phys. Rev. B 76, 054303 (2007).

${ }^{11}$ M.-H. Lu, X.-K. Liu, L. Feng, J. Li, C.-P. Huang, Y.-F. Chen, Y.-Y. Zhu, S.-N. Zhu, and N.-B. Ming, Phys. Rev. Lett. 99, 174301 (2007).

${ }^{12}$ J. Christensen, L. Martin-Moreno, and F. J. Garcia-Vidal, Phys. Rev. Lett. 101, 014301 (2008).

${ }^{13}$ H. Estrada, P. Candelas, A. Uris, F. Belmar, F. Meseguer, and F. J. García de Abajo, Appl. Phys. Lett. 93, 011907 (2008).

${ }^{14}$ F. J. García de Abajo, H. Estrada, and F. J. Meseguer, New J. Phys. 11, 093013 (2009). 